International Journal of Pure and Applied Mathematics

Volume 92 No. 3 2014, 305-334

ISSN: 1311-8080 (printed version); ISSN: 1314-3395 (on-line version)

url: http://www.ijpam.eu

doi: http://dx.doi.org/10.12732/ijpam.v92i3.1

\title{
ON A PERTURBED SYSTEM OF CHEMOTAXIS, II
}

\author{
Masaki Kurokiba $^{1 \S}$, Takashi Suzuki², Ryo Takahashi ${ }^{3}$ \\ ${ }^{1}$ Mathematical Science Rearch Unit \\ Muroran Institute of Technology \\ 27-1, Mizumoto Muroran, Hokkaido, 050-8585, JAPAN \\ ${ }^{2,3}$ Division of Mathematical Science \\ Graduate School of Engineering Science \\ Osaka University \\ Toyonaka, 560-8531, JAPAN
}

\begin{abstract}
We study a parabolic-elliptic system with nonlinear boundary condition, describing the chemotactic aggregation of cellular slime molds. We show the well-posedness locally in time, blowup criterion of the solution, and finiteness of the blowup points.
\end{abstract}

AMS Subject Classification: 35K55, 35K57, 92C15, 92D15

Key Words: chemotaxis, parabolic-elliptic, nonlinear boundary condition, blowup solution

\section{Introduction}

This paper is concerned with the parabolic-elliptic system describing chemo-

Received: August 12, 2011

(C) 2014 Academic Publications, Ltd. url: www.acadpubl.eu

${ }^{\S}$ Correspondence author 
tactic aggregation, that is,

$$
\begin{cases}u_{t}=\nabla \cdot(\nabla u-\chi u \nabla v) & \text { in } \Omega \times(0, T) \\ 0=\Delta v-\gamma v-f(v)+\alpha u & \text { in } \Omega \times(0, T) \\ \frac{\partial u}{\partial \nu}-\chi u \frac{\partial v}{\partial \nu}=0 & \text { on } \partial \Omega \times(0, T) \\ \frac{\partial v}{\partial \nu}+g(v)=0 & \text { on } \partial \Omega \times(0, T) \\ \left.u\right|_{t=0}=u_{0} & \text { on } \bar{\Omega},\end{cases}
$$

where $\Omega$ is a bounded domain in $\boldsymbol{R}^{n}$ with smooth boundary $\partial \Omega, \chi, \alpha, \gamma$ are positive constants, $f=f(s), g=g(s)$ are smooth functions, and $\nu$ is the unit outward normal vector on $\partial \Omega$. The unknown functions $u=u(x, t)$ and $v=v(x, t)$ stand for the cell density and the concentration of the chemical substance at $(x, t) \in \bar{\Omega} \times[0, T)$, respectively, and $F=-\nabla u+\chi u \nabla v$ is the flux of $u$ so that the effect of diffusion $-\nabla \cdot \nabla u$ and that of chemotaxis compete for $\mathrm{u}$ to vary. Another description is the movement from the gravitational equilibrium of polytropic fluid, see [4], [6].

For the problem (GCZ) with $f(v)=g(v)=0$, Nagai [16] showed that the conjecture of Childress and Percus [9] concerning $n=2$ is true for radially symmetric solutions, that is, the chemotaxis collapse can occur if the total cell number on $\Omega \subset \boldsymbol{R}^{2}$ is larger than $8 \pi / \alpha \chi$ and can not occur in the other case, and then, Nagai, Senba and Yosida [17] and Senba and Suzuki [22] corrected this value to $4 \pi / \alpha \chi$ in the general case, see also Biler [5] and Gajewski and Zacharias [11]. Then, Senba and Suzuki [21] and Suzuki [23] showed the formation of collapses with the quantized mass for the blowup solution in finite time, refining the conjecture of Nanjundiah [19].

For the problem (GCZ) with $f(v)=\beta|v|^{p-1} v$ and $g(v)=0$, Chen and Zhong [8] studied the existence and the non-existence of the solution global in time, while Kurokiba and Suzuki [13] showed the finiteness of the blowup points of the blowup solution in finite time.

In this paper we impose the following conditions on (GCZ):

$$
\begin{array}{r}
u_{0} \geq 0, u_{0} \not \equiv 0 \text { on } \Omega \\
u_{0} \in C^{3}(\bar{\Omega}) \\
f, g \text { is smooth, say } C^{2} \\
f^{\prime}(s), g^{\prime}(s) \geq 0 \\
f(0), g(0) \leq 0 \\
\left|f^{\prime}(s)\right| \leq C\left(1+|s|^{p_{1}-1}\right) \\
\left|g^{\prime}(s)\right| \leq C\left(1+|s|^{p_{2}-1}\right)
\end{array}
$$


for all $s \in \boldsymbol{R}$, where $C>0, p_{1} \in\left(1, p^{*}\right)$ and $p_{2} \in\left(1, p^{* *}\right)$ with

$$
\begin{aligned}
p^{*} & = \begin{cases}\infty & \text { if } n=2 \\
\frac{n+2}{n-2} & \text { if } n \geq 3\end{cases} \\
p^{* *} & = \begin{cases}\infty & \text { if } n=2 \\
\frac{n}{n-2} & \text { if } n \geq 3\end{cases}
\end{aligned}
$$

We assume the compatibility condition to the parabolic part:

$$
\frac{\partial u_{0}}{\partial \nu}-u_{0} \frac{\partial v_{0}}{\partial \nu}=0,
$$

where $v_{0}$ denotes the solution to (2.1) for $u=u_{0}$, see the following section. Then, we obtain the classical solution local in time:

Theorem 1. If (1.1)-(1.9) hold, then there exists a unique non-negative classical solution $(u, v) \in C^{2+\theta, 1+\theta / 2}\left(\overline{Q_{T}}\right) \times C\left([0, T] ; C^{2+\theta}(\bar{\Omega})\right)$ to $(\mathrm{GCZ})$ with $\theta \in(0,1)$ provided that $T$ is sufficiently small.

Henceforth $T_{\max } \in(0,+\infty]$ and $\mathcal{B}$ stand for the maximal existence time of the solution and the blowup set of $u$, respectively:

$$
\begin{gathered}
T_{\max }=\sup \{T>0 \mid \text { the solution }(u(\cdot, t), v(\cdot, t)) \text { exists for } t \in[0, T)\}, \\
\mathcal{B}=\left\{x_{0} \in \bar{\Omega} \mid \text { there exist } t_{k} \uparrow T_{\max }\right. \\
\text { and } \left.x_{k} \rightarrow x_{0} \text { such that } u\left(x_{k}, t_{k}\right) \rightarrow+\infty \text { as } k \rightarrow \infty\right\},
\end{gathered}
$$

and call each $x_{0} \in \mathcal{B}$ the blowup point. The following theorem assures that $T_{\max }<+\infty$ implies $\mathcal{B} \neq \emptyset$ :

Theorem 2. If (1.1)-(1.9), $n=2$ and $T_{\max }<+\infty$ hold, then it holds that

$$
\lim _{t \uparrow T_{\max }} \int_{\Omega} u \log u d x=\infty .
$$

Now, we state the main theorem.

Theorem 3. If (1.1)-(1.9), $n=2$ and $T_{\max }<+\infty$ hold, then it holds that $\sharp(\mathcal{B} \cap \Omega)<+\infty$.

Concerning the finiteness of $\mathcal{B} \cap \partial \Omega$, see the final remark of this paper.

This paper is composed of four sections. Theorems 1,2 , and 3 are proven in sections 2,3 , and 4 , respectively. Henceforth, we put $\chi=\alpha=\gamma=1$ without loss of generality. 


\section{Proof of Theorem 1}

To begin with, we study the elliptic equation

$$
\begin{cases}-\Delta v+v+f(v)=u & \text { in } \Omega \\ \frac{\partial v}{\partial \nu}+g(v)=0 & \text { on } \partial \Omega .\end{cases}
$$

Lemma 4. If (1.3)-(1.7) hold and $u=u(x) \geq 0$ is in $C^{1}(\bar{\Omega})$, then (2.1) has a unique non- negative classical solution $v=v(x) \in C^{2+\theta}(\bar{\Omega})$.

Proof. To confirm the uniqueness of $v$, let $v_{1}$ and $v_{2}$ be the classical solutions to $(2.1)$ and set $w:=v_{1}-v_{2}$. Then, it holds that

$$
\begin{cases}-\Delta w+w=-f\left(v_{1}\right)+f\left(v_{2}\right) & \text { in } \Omega \\ \frac{\partial w}{\partial \nu}=-g\left(v_{1}\right)+g\left(v_{2}\right) & \text { on } \partial \Omega\end{cases}
$$

and therefore,

$$
\begin{aligned}
\int_{\Omega}|\nabla w|^{2}+w^{2} d x= & -\int_{\partial \Omega}\left(v_{1}-v_{2}\right)\left(g\left(v_{1}\right)-g\left(v_{2}\right)\right) d S \\
& -\int_{\Omega}\left(v_{1}-v_{2}\right)\left(f\left(v_{1}\right)-f\left(v_{2}\right)\right) d x \leq 0
\end{aligned}
$$

by (1.4). This implies $v_{1}=v_{2}$.

To show the non-negativity $v \geq 0$, we take $v_{-}=\min (v, 0) \leq 0$. Since

$$
\int_{\Omega}\left|\nabla v_{-}\right|^{2}+v_{-}^{2}+f(v) v_{-} d x+\int_{\partial \Omega} g(v) v_{-} d S=\int_{\Omega} u v_{-} d x \leq 0
$$

holds by (2.1), it follows that $v_{-}=0$ from (1.4)-(1.5).

The existence of $v$ is obtained by the variational method. We set

$$
I[v]:=\frac{1}{2} \int_{\Omega}|\nabla v|^{2}+v^{2} d x-\int_{\Omega} u v d x+\int_{\Omega} \tilde{f}\left(v_{+}\right) d x+\int_{\partial \Omega} \tilde{g}\left(v_{+}\right) d S
$$

for $v_{+}=\max (v, 0) \geq 0$ and

$$
\tilde{f}(v):=\int_{0}^{v} f(s) d s, \quad \tilde{g}(v):=\int_{0}^{v} g(s) d s .
$$

and show that $I$ attains the minimum in $H^{1}=H^{1}(\Omega)$, using (1.6)-(1.7). Since the assumption (1.6)-(1.7) guarantees the sub-criticalness of the nonlinearity, the minimizer will actually be in $C^{2+\theta}(\bar{\Omega})$ by the bootstrap argument. 
First, we confirm the coercivity of the functional $I$. In fact, given $0<\delta \ll 1$, we take $L=L(\delta)>0$, using (1.4), such that

$$
\frac{f(s)}{s} \geq \frac{f(0)}{s} \geq-\delta, \quad \frac{g(s)}{s} \geq \frac{g(0)}{s} \geq-\delta \quad \text { for all } s \geq L .
$$

It holds that

$$
\begin{aligned}
\tilde{f}(v) & =\int_{0}^{L} f(s) d s+\int_{L}^{v} \frac{f(s)}{s} \cdot s d s \\
& \geq \int_{0}^{L} f(s) d s+\frac{\delta}{2} L^{2}-\frac{\delta}{2} v^{2} \geq-\delta v^{2}
\end{aligned}
$$

for $v \geq l_{1}$, where $l_{1}=\max \left\{L, \sqrt{2 \delta^{-1}\left|\int_{0}^{L} f(s) d s+\delta L^{2} / 2\right|}\right\}$, and similarly,

$$
\tilde{g}(v) \geq-\delta v^{2}
$$

for $v \geq l_{2}$, where $l_{2}=\max \left\{L, \sqrt{2 \delta^{-1}\left|\int_{0}^{L} g(s) d s+\delta L^{2} / 2\right|}\right\}$. Therefore,

$$
\begin{aligned}
& \int_{\Omega} \tilde{f}\left(v_{+}\right) d x+\int_{\partial \Omega} \tilde{g}\left(v_{+}\right) d S=\int_{\left\{0 \leq v<l_{1}\right\}} \tilde{f}(v) d x+\int_{\left\{v \geq l_{1}\right\}} \tilde{f}(v) d x \\
& \quad+\int_{\left\{0 \leq v<l_{2}\right\}} \tilde{g}(v) d S+\int_{\left\{v \geq l_{2}\right\}} \tilde{g}(v) d S \\
& \geq-\delta \int_{\Omega} v^{2} d x-\delta \int_{\partial \Omega} v^{2} d S-C(\Omega, f, g)
\end{aligned}
$$

where $C(\Omega, f, g)=|\Omega| \min _{|s| \leq l_{1}} \tilde{f}(s)+|\partial \Omega| \min _{|s| \leq l_{2}} \tilde{g}(s)$.

Since

$$
\int_{\Omega}|\nabla v|^{2} d x+\int_{\Omega} v^{2} d x \geq \sigma \int_{\partial \Omega} v^{2} d S
$$

holds for some constant $\sigma=\sigma(n, \Omega)>0$ by the continuity of the trace operator $\gamma: H^{1}(\Omega) \rightarrow L^{2}(\partial \Omega)$, we obtain

$$
\begin{aligned}
I[v] \geq & \frac{1}{2} \int_{\Omega}|\nabla v|^{2} d x+\left(\frac{1}{2}-\varepsilon-\delta\right) \int_{\Omega} v^{2} d x-\delta \int_{\partial \Omega} v^{2} d S \\
& -\frac{1}{4 \varepsilon} \int_{\Omega} u^{2} d x-C(\Omega, f, g) \\
\geq & \left(\frac{1}{2}-\sigma^{-1} \delta\right) \int_{\Omega}|\nabla v|^{2} d x+\left(\frac{1}{2}-\varepsilon-\delta-\sigma^{-1} \delta\right) \int_{\Omega} v^{2} d x
\end{aligned}
$$




$$
\begin{aligned}
& -\frac{1}{4 \varepsilon} \int_{\Omega} u^{2} d x-C(\Omega, f, g) \\
\geq & \left(\frac{1}{2}-\varepsilon-\delta-\sigma^{-1} \delta\right)\|v\|_{H^{1}}^{2}-\frac{1}{4 \varepsilon} \int_{\Omega} u^{2} d x-C(\Omega, f, g)
\end{aligned}
$$

by (2.2) and (2.4)-(2.5), where $\varepsilon>0$ is arbitrary. Taking $\delta, \varepsilon>0$ such that $\left(\frac{1}{2}-\varepsilon-\delta-\sigma^{-1} \delta\right)>0$, we thus obtain the coerciveness of $I$ on $H^{1}(\Omega)$ :

$$
I[v] \geq \nu\|v\|_{H^{1}}^{2}-C, \quad v \in H^{1}(\Omega),
$$

where $\nu>0$ and $C>0$ are constants.

To confirm the weak lower semi-continuity of $I$, let $\left\{v_{k}\right\} \subset H^{1}(\Omega)$ be a minimizing sequence:

$$
I\left[v_{k}\right] \rightarrow \inf _{w \in H^{1}} I[w]>-\infty .
$$

Since $\left\{v_{k}\right\}$ is bounded from below by (2.6), we can extract a subsequence, denoted by the same symbol, such that

$$
v_{k} \rightarrow v \text { weakly in } H^{1}(\Omega)
$$

for some $v$, and then it follows that

$$
\|v\|_{H^{1}} \leq \liminf _{k}\left\|v_{k}\right\|_{H^{1}} .
$$

Meanwhile, we have the compact imbedding

$$
H^{1}(\Omega) \hookrightarrow W^{s, q}(\Omega)
$$

and the continuous imbedding

$$
W^{s, q}(\Omega) \hookrightarrow W^{s-\frac{1}{q}, q}(\partial \Omega)
$$

for $0<s<1$ and $1 \leq q<q^{*}, \frac{1}{q^{*}}=\frac{1}{2}-\frac{1-s}{n}$, see [1], which implies the compact imbeddings

$$
H^{1}(\Omega) \hookrightarrow L^{p_{1}+1}(\Omega), L^{p_{2}+1}(\partial \Omega)
$$

for $p_{1}, p_{2}$ prescribed by (1.8). From this compactness, passing if necessary to yet another subsequence, we see that there exist $h_{1} \in L^{p_{1}+1}(\Omega)$ and $h_{2} \in L^{p_{2}+1}(\partial \Omega)$ such that

$$
\begin{aligned}
& v_{k}(x) \rightarrow v(x) \text { for a.e. } x \in \Omega, \quad v_{k}(\xi) \rightarrow v(\xi) \text { for a.e. } \xi \in \partial \Omega, \\
& \left|v_{k}(x)\right| \leq h_{1}(x) \text { for a.e. } x \in \Omega, \quad\left|v_{k}(\xi)\right| \leq h_{2}(\xi) \text { for a.e. } \xi \in \partial \Omega .
\end{aligned}
$$


Since $\tilde{f}$ and $\tilde{g}$ are continuous

$$
\tilde{f}\left(v_{k}(x)\right) \rightarrow \tilde{f}\left(v_{k}(x)\right) \text { for a.e. } x \in \Omega, \quad \tilde{g}\left(v_{k}(\xi)\right) \rightarrow \tilde{g}\left(v_{k}(\xi)\right) \text { for a.e. } \xi \in \Omega,
$$

and it follows from (1.6)-(1.7) that

$$
\begin{aligned}
& \left|\tilde{f}\left(v_{k}(x)\right)\right| \leq C\left(1+h_{1}^{p_{1}+1}(x)\right) \text { for a.e. } x \in \Omega, \\
& \left|\tilde{g}\left(v_{k}(\xi)\right)\right| \leq C\left(1+h_{2}^{p_{2}+1}(\xi)\right) \text { for a.e. } \xi \in \Omega .
\end{aligned}
$$

Then we have

$$
\begin{aligned}
& -\int_{\Omega} u v_{k} d x+\int_{\Omega} \tilde{f}\left(v_{k}\right) d x+\int_{\partial \Omega} \tilde{g}\left(v_{k}\right) d S \\
& \longrightarrow-\int_{\Omega} u v d x+\int_{\Omega} \tilde{f}(v) d x+\int_{\partial \Omega} \tilde{g}(v) d S
\end{aligned}
$$

by the Lebesgue dominated convergence theorem together with the above facts, and consequently

$$
I[v] \leq \liminf _{k} I\left[v_{k}\right] .
$$

We obtain $I[v]=\inf _{w \in H^{1}} I[w]$ as desired.

Finally, we show the regurality of $v$. We shall do it only in the case $n \geq 3$, for it is done more easily in the case $n=2$. Set

$$
r=\frac{2 n}{p_{1}(n-2)} \in\left(\frac{2 n}{n+2}, \frac{2 n}{n-2}\right), \quad s=\frac{2 n}{n+(n-2)\left(p_{2}-1\right)} \in\left(\frac{2 n}{n+2}, 2\right) .
$$

We use (1.6)-(1.7) to get

$$
\|u-f(v)\|_{r} \leq\|u\|_{r}+C\left(1+\|v\|_{r p_{1}}^{p_{1}}\right) \leq\|u\|_{r}+C\left(1+\|v\|_{H^{1}}^{p_{1}}\right)
$$

and

$$
\begin{aligned}
\|g(v)\|_{W^{1-1 / s, s}(\partial \Omega)} & \leq C\left(\|g(v)\|_{s}+\left\|g^{\prime}(v) \mid \nabla v\right\|_{s}\right) \\
& \leq C\left(1+\|v\|_{p_{2} s}^{p_{2}}+\left\|g^{\prime}(v)\right\|_{\frac{2 s}{2-s}}\|\nabla v\|_{2}\right) \\
& \leq C\left(1+\|v\|_{H^{1}}^{p_{2}}+\|\nabla v\|_{2}+\|v\|_{\frac{2 s\left(p_{2}-1\right)}{2-s}}^{p_{2}}\|\nabla v\|_{2}\right) \\
& =C\left(1+\|v\|_{H^{1}}^{p_{2}}+\|\nabla v\|_{2}+\|v\|_{\frac{2 n}{n-2}}^{p_{2}-1}\|\nabla v\|_{2}\right) \\
& \leq C\left(1+\|v\|_{H^{1}}+\|v\|_{H^{1}}^{p_{2}}\right) .
\end{aligned}
$$

From these inequalities and the $L^{p}$ estimates (see [2]), it follows that

$$
v \in W^{2, \tilde{q_{0}}}(\Omega) \quad \text { with } \tilde{q_{0}}=\min (r, s) \in\left(\frac{2 n}{n+2}, 2\right) .
$$


If $2 n /(n+2)<\tilde{q}_{k-1} \leq n$, then we define $q_{k}, r_{k}, s_{k}, \tilde{q}_{k}$ recursively as follows:

$$
\begin{gathered}
q_{k}= \begin{cases}\frac{n \tilde{q}_{k-1}}{n-\tilde{q}_{k-1}} & \left(\frac{2 n}{n+2}<\tilde{q}_{k-1}<n\right) \\
\text { any larger real than } n & \left(\tilde{q}_{k-1}=n\right),\end{cases} \\
r_{k}= \begin{cases}\frac{q_{k}^{*}}{p_{1}} & \left(\frac{2 n}{n+2}<\tilde{q}_{k-1}<\frac{n}{2}\right) \\
\text { any larger real than } n & \left(\frac{n}{2} \leq \tilde{q}_{k-1} \leq n\right),\end{cases} \\
s_{k}= \begin{cases}\frac{q_{k} q_{k}^{*}}{q_{k}^{*}+q_{k}\left(p_{2}-1\right)} & \left(\frac{2 n}{n+2}<\tilde{q}_{k-1}<\frac{n}{2}\right) \\
\text { any real in }\left(\frac{n}{2}, n\right) & \left(\tilde{q}_{k-1}=\frac{n}{2}\right) \\
\text { any real in }\left(n, q_{k}\right) & \left(\frac{n}{2}<\tilde{q}_{k-1} \leq n\right),\end{cases}
\end{gathered}
$$

where $q_{k}^{*}=n q_{k} /\left(n-q_{k}\right)$ for $\tilde{q}_{k-1} \in(2 n /(n+2), n / 2)$. For the above $r_{k}$ and $s_{k}$, we similarly deduce

$$
\begin{aligned}
& \|u-f(v)\|_{r_{k}} \leq\|u\|_{r_{k}}+C\left(1+\|v\|_{W^{1, q_{k}}}^{p_{1}}\right) \\
& \|g(v)\|_{W^{1-1 / s_{k}, s_{k}}(\partial \Omega)} \leq C\left(1+\|v\|_{W^{1, q_{k}}}+\|v\|_{W^{1, q_{k}}}^{p_{2}}\right) .
\end{aligned}
$$

Then we use the $L^{p}$ estimates, again, so that $v \in W^{2, \tilde{q}_{k}}(\Omega)$. Repeating this finite times, we obtain

$$
v \in W^{2, l}(\Omega) \hookrightarrow C^{2-n / l}(\bar{\Omega}) \quad \text { for some } l>n,
$$

and conclude the desired regularity $v \in C^{2+\theta}(\bar{\Omega})$ by the Schauder estimate, see [2]. The proof is complete.

Proof of Theorem 1. We define

$$
u^{(0)} \equiv u_{0} \quad \text { on } \bar{\Omega},
$$

and $v^{(0)}, u^{(1)}, v^{(1)}, \cdots$ inductively as follows:

$$
\begin{cases}-\Delta v^{(k)}+v^{(k)}=-f\left(v^{(k)}\right)+u^{(k)} & \text { in } Q_{T} \\ \frac{\partial v^{(k)}}{\partial \nu}=-g\left(v^{(k)}\right) & \text { on } \Gamma_{T}\end{cases}
$$

and

$$
\begin{cases}u_{t}^{(k+1)}-\Delta u^{(k+1)}+\nabla \cdot\left(u^{(k+1)} \nabla v_{0}\right)=-\nabla \cdot\left(u^{(k)} \nabla\left(v^{(k)}-v_{0}\right)\right) & \text { in } Q_{T} \\ \frac{\partial u^{(k+1)}}{\partial \nu}-\frac{\partial v_{0}}{\partial \nu} u^{(k+1)}=\left(\frac{\partial v^{(k)}}{\partial \nu}-\frac{\partial v_{0}}{\partial \nu}\right) u^{(k)} & \text { in } \Gamma_{T} \\ \left.u^{(k+1)}\right|_{t=0}=u_{0} & \text { on } \bar{\Omega}\end{cases}
$$


where $Q_{T}:=\Omega \times(0, T)$ and $\Gamma_{T}:=\partial \Omega \times(0, T)$. We take

$$
q>n+2
$$

and obtain the continuous imbedding

$$
W_{q}^{2,1}\left(Q_{T}\right) \hookrightarrow C^{2-(n+2) / q, 1-(n+2) / 2 q}\left(\overline{Q_{T}}\right),
$$

where $C^{l, l / 2}\left(\overline{Q_{T}}\right)(0<l \notin \boldsymbol{N})$ is the standard Hölder space and its norm is denoted by $[\cdot]_{l, Q_{T}}$, see $[14]$.

Set

$$
X_{M, T}:=\left\{u \in W_{q}^{2,1}\left(Q_{T}\right)\left|u \geq 0,\|u\|_{W_{q}^{2,1}\left(Q_{T}\right)} \leq M, \quad u\right|_{t=0}=u_{0}\right\}
$$

for $M>0$ and $0<T \ll 1$ to be decided later. From (2.11), (2.12) and Lemma 4 , we can define $v^{(k)}$ as the solution to (2.9) with non-negativity if $u^{(k)} \in X_{M, T}$. Henceforth, $C_{i}^{*}(i=1,2, \cdots)$ denotes the positive constant which is monotone increasing on $T$ and depends on $M$ and given data, but not on $u^{(k)}$ and $v^{(k)}$. Using the mean value theorem and standard elliptic regularity, we can find the inequality

$$
\sup _{\substack{x \in \bar{\Omega} \\ t, s \in[0, T], t \neq s}} \frac{\left|v^{(k)}(x, t)-v^{(k)}(x, s)\right|+\left|\nabla v^{(k)}(x, t)-\nabla v^{(k)}(x, s)\right|}{|t-s|^{1-(n+2) / 2 q}} \leq C_{1}^{*},
$$

where $C_{1}^{*}$ is independent of $T$.

If $u^{(k)} \in X_{M, T}$ and $v^{(k)}$ is a solution to (2.9), then there exists a unique solution $u^{(k+1)} \in W_{q}^{2,1}\left(Q_{T}\right)$ to $(2.10)$ such that

$$
\begin{aligned}
\left\|u^{(k+1)}\right\|_{W_{q}^{2,1}\left(Q_{T}\right)} \leq & C_{0}^{*}(T)\left\{\left\|u_{0}\right\|_{W^{2, q}(\Omega)}+\left\|\nabla \cdot\left(u^{(k)} \nabla\left(v^{(k)}-v_{0}\right)\right)\right\|_{L^{q}\left(Q_{T}\right)}\right. \\
& \left.+\left\|\left(\frac{\partial v^{(k)}}{\partial \nu}-\frac{\partial v_{0}}{\partial \nu}\right) u^{(k)}\right\|_{W_{q}^{1-1 / q, 1 / 2-1 / 2 q}\left(\Gamma_{T}\right)}\right\}
\end{aligned}
$$

by the $W_{q}^{2,1}$-theory, see [14]. Note that $C_{0}^{*}(T)$ is independent of $M$ and monotone increasing on $T$. We compute

$$
\begin{aligned}
& \left\|\nabla \cdot\left(u^{(k)} \nabla\left(v^{(k)}-v_{0}\right)\right)\right\|_{L^{q}\left(Q_{T}\right)} \\
\leq \quad M( & \left\|v^{(k)}-v_{0}\right\|_{L^{\infty}\left(Q_{T}\right)}+\left\|\nabla\left(v^{(k)}-v_{0}\right)\right\|_{L^{\infty}\left(Q_{T}\right)} \\
& \left.\quad+\left\|f\left(v^{(k)}\right)-f\left(v_{0}\right)\right\|_{L^{\infty}\left(Q_{T}\right)}+\left\|u^{(k)}-u_{0}\right\|_{L^{\infty}\left(Q_{T}\right)}\right) .
\end{aligned}
$$


It holds that

$$
\left\|v^{(k)}-v_{0}\right\|_{C\left(\overline{Q_{T}}\right)}+\left\|\nabla\left(v^{(k)}-v_{0}\right)\right\|_{C\left(\overline{Q_{T}}\right)} \leq T^{1-(n+2) / 2 q} C_{1}^{*}
$$

by (2.14). Since $f$ is smooth, we have

$$
\begin{aligned}
\left\|f\left(v^{(k)}\right)-f\left(v_{0}\right)\right\|_{L^{\infty}\left(Q_{T}\right)} & \leq C_{2}^{*}\left\|v^{(k)}-v_{0}\right\|_{L^{\infty}\left(Q_{T}\right)} \\
& \leq T^{1-(n+2) / 2 q} C_{1}^{*} C_{2}^{*} .
\end{aligned}
$$

We remark the following fact (see [14]): If (2.11) holds and $0<T \ll 1$ then

$$
[u]_{1+\theta, Q_{T}} \leq C_{3}^{*}\left(T^{\theta / 2}\|u\|_{W_{q}^{2,1}\left(Q_{T}\right)}+T^{-1+\theta / 2}\|u\|_{L^{q}\left(Q_{T}\right)}\right)
$$

where $\theta=1-(n+2) / q$ and the constant $C_{3}^{*}$ is independent of $0<T \ll 1$. Using Hölder's inequality, we have

$$
\left\|u^{(k)}-u_{0}\right\|_{L^{q}\left(Q_{T}\right)}^{q} \leq \frac{1}{q} T^{q}\left\|u_{t}^{(k)}\right\|_{L^{q}\left(Q_{T}\right)}^{q},
$$

and then

$$
\left[u^{(k)}-u_{0}\right]_{1+\theta, Q_{T}} \leq T^{\theta / 2} \cdot 3 C_{3}^{*} M
$$

by (2.19). Combining (2.16)-(2.18) and (2.20), we obtain

$$
\left\|\nabla \cdot\left(u^{(k)} \nabla\left(v^{(k)}-v_{0}\right)\right)\right\|_{L^{q}\left(Q_{T}\right)} \leq C_{4}^{*} T^{1 / 2-(n+2) / 2 q} .
$$

Next, we estimate the boundary integral term as

$$
\begin{aligned}
& \left\|\left(\frac{\partial v^{(k)}}{\partial \nu}-\frac{\partial v_{0}}{\partial \nu}\right) u^{(k)}\right\|_{W_{q}^{1-1 / q, 1 / 2-1 / 2 q}\left(\Gamma_{T}\right)} \\
\leq & \left\|g\left(v^{(k)}\right)-g\left(v_{0}\right)\right\|_{L^{\infty}\left(\Gamma_{T}\right)} \cdot\left\|u^{(k)}\right\|_{W_{q}^{1-1 / q, 1 / 2-1 / 2 q}\left(\Gamma_{T}\right)} \\
& +\left\|g\left(v^{(k)}\right)-g\left(v_{0}\right)\right\|_{W_{q}^{1-1 / q, 1 / 2-1 / 2 q}\left(\Gamma_{T}\right)} \cdot\left\|u^{(k)}\right\|_{L^{\infty}\left(\Gamma_{T}\right)} \\
\leq & C M\left(\left\|g\left(v^{(k)}\right)-g\left(v_{0}\right)\right\|_{L^{\infty}\left(\Gamma_{T}\right)}+\left\|g\left(v^{(k)}\right)-g\left(v_{0}\right)\right\|_{W_{q}^{1-1 / q, 1 / 2-1 / 2 q}\left(\Gamma_{T}\right)}\right),
\end{aligned}
$$

where $\mathrm{C}$ is independent of $\mathrm{M}$ and $\mathrm{T}$. Since $\mathrm{g}$ is smooth and (2.14) holds, we have

$$
\left\|g\left(v^{(k)}\right)-g\left(v_{0}\right)\right\|_{L^{\infty}\left(\Gamma_{T}\right)} \leq T^{1-(n+2) / 2 q} C_{5}^{*}
$$

Following the notations of [14], we take the canonical set $B \subset \boldsymbol{R}^{n-1}$, for instance the unit cube in $\boldsymbol{R}^{n-1}$, and the finite family of the mappings $\Phi_{j}: B \rightarrow$ 
$V_{j} \subset \partial \Omega(j \in \mathcal{J})$ each of which is one-to-one, onto and smooth, and satisfies $\cup_{j \in \mathcal{J}} \Phi_{j} B=\partial \Omega$. Let $\left\{\rho_{j}\right\}_{j \in \mathcal{J}}, 0 \leq \rho_{j} \leq 1$, be a smooth partition of unity subject to $\left\{\Phi_{j}(B)\right\}_{j \in \mathcal{J}}$. Set

$$
\|u\|_{W_{q}^{\lambda, \lambda / 2}\left(\Gamma_{T}\right)}:=\|u\|_{L^{q}\left(\Gamma_{T}\right)}+<u>_{L_{q}^{\lambda, \lambda / 2}\left(\Gamma_{T}\right)}
$$

for $0<\lambda<1$, where

$$
\begin{aligned}
\|u\|_{L^{q}\left(\Gamma_{T}\right)}:= & \sum_{j \in \mathcal{J}}\left\|\Phi_{j}^{*}\left(u \rho_{j}\right)\right\|_{L^{q}(B \times(0, T))}, \\
\left\langle u>_{L_{q}^{\lambda, \lambda / 2}\left(\Gamma_{T}\right)}:=\right. & \sum_{j \in \mathcal{J}}<\Phi_{j}^{*}\left(u \rho_{j}\right)>_{L_{q}^{\lambda, \lambda / 2}(B \times(0, T))}, \\
<w>_{L_{q}^{\lambda, \lambda / 2}(B \times(0, T))}:= & \left\{\int_{0}^{T} d t \iint_{B \times B} \frac{|w(x, t)-w(y, t)|^{q}}{|x-y|^{n-1+\lambda q}} d x d y\right\}^{1 / q} \\
& +\left\{\int_{B} d x \int_{0}^{T} \int_{0}^{T} \frac{|w(x, t)-w(x, s)|^{q}}{|t-s|^{1+\lambda q / 2}} d t d s\right\}^{1 / q} \\
=: & <w>_{L_{q}^{\lambda, 0}(B \times(0, T))}+<w>_{L_{q}^{0, \lambda / 2}(B \times(0, T))},
\end{aligned}
$$

and $\Phi_{j}^{*}$ is the pull back induced by $\Phi_{j}$.

We set $w_{j}^{(k)}=\Phi_{j}^{*}\left(v^{(k)} \rho_{j}\right)$ and may take the unit cube as the above $B$. We calculate according to the above definition and get

$$
\begin{aligned}
&\left\|v^{(k)}\right\|_{L^{q}\left(\Gamma_{T}\right)}+<v^{(k)}>_{L_{q}^{1-1 / q, 0}\left(\Gamma_{T}\right)} \leq T^{1 / q} C \sup _{0<t<T}\left\|v^{(k)}(\cdot, t)\right\|_{W^{1, q}(\Omega)}, \\
&<w_{j}^{(k)}>_{L_{q}^{0,(1-1 / q) / 2}(B \times(0, T))} \leq T^{(q+1-n) / 2 q} C_{1}^{*} .
\end{aligned}
$$

From the elliptic regularity and (2.20) we have

$$
\begin{aligned}
\left\|v^{(k)}(t)-v_{0}\right\|_{W^{1, q}(\Omega)} & \leq C_{6}^{*}\left\|u^{(k)}(t)-u_{0}\right\|_{C(\bar{\Omega})} \\
& \leq T^{\frac{1}{2}(1-(n+2) / q)} \cdot 3 C_{3}^{*} C_{6}^{*} M
\end{aligned}
$$

and then

$$
\sup _{0<t<T}\left\|v^{(k)}(\cdot, t)\right\|_{W^{1, q}(\Omega)} \leq\left\|v_{0}\right\|_{W^{1, q}}+T^{\frac{1}{2}(1-(n+2) / q)} \cdot 2 C_{3}^{*} C_{6}^{*} M .
$$

Combining (2.24), (2.25) and (2.27), we get

$$
\left\|v^{(k)}\right\|_{W_{q}^{1-1 / q, 1 / 2-1 / 2 q}\left(\Gamma_{T}\right)} \leq T^{1 / q} C_{7}^{*}
$$


One can see that if there exist constants $A_{i}(i=1,2)$ such that

$$
\begin{array}{r}
\left\|v^{(k)}\right\|_{L^{\infty}\left(\Gamma_{T}\right)}+\left\|v_{0}\right\|_{L^{\infty}\left(\Gamma_{T}\right)} \leq A_{1}, \\
<v^{(k)}>_{L_{q}^{1-1 / q, 1 / 2-1 / 2 q}\left(\Gamma_{T}\right)}+<v_{0}>_{L_{q}^{1-1 / q, 1 / 2-1 / 2 q}\left(\Gamma_{T}\right)} \leq A_{2},
\end{array}
$$

then there exists a constant $C_{8}^{*}$ depending only on $q, A_{1}, A_{2}, g$ and $\Omega$ such that

$$
\begin{aligned}
& \left\|g\left(v^{(k)}\right)-g\left(v_{0}\right)\right\|_{W_{q}^{1-1 / q, 1 / 2-1 / 2 q}\left(\Gamma_{T}\right)} \\
& \leq C_{8}^{*}\left(\left\|v^{(k)}-v_{0}\right\|_{W_{q}^{1-1 / q, 1 / 2-1 / 2 q}\left(\Gamma_{T}\right)}+\left\|v^{(k)}-v_{0}\right\|_{L^{\infty}\left(\Gamma_{T}\right)}\right) .
\end{aligned}
$$

It holds that

$$
\begin{aligned}
& \left\|v^{(k)}-v_{0}\right\|_{L^{q}\left(\Gamma_{T}\right)}+<v^{(k)}-v_{0}>{ }_{L_{q}^{1-1 / q, 0}\left(\Gamma_{T}\right)} \\
\leq & T^{1 / q} C \sup _{0<t<T}\left\|v^{(k)}(\cdot, t)-v_{0}\right\|_{W^{1, q}(\Omega)} \\
\leq & T^{\frac{1}{2}(1-n / q)} C_{9}^{*}
\end{aligned}
$$

by (2.26) and the trace embedding, and that

$$
\begin{aligned}
<v^{(k)}-v_{0}>_{L_{q}^{0,1 / 2-1 / 2 q}\left(\Gamma_{T}\right)} & =\left\langle v^{(k)}>_{L_{q}^{0,1 / 2-1 / 2 q}\left(\Gamma_{T}\right)}\right. \\
& \leq T^{(q+1-n) / 2 q} C_{1}^{* \sharp \mathcal{J}}
\end{aligned}
$$

by $(2.25)$. Hence, we get

$$
\left\|v^{(k)}-v_{0}\right\|_{W_{q}^{1-1 / q, 1 / 2-1 / 2 q}\left(\Gamma_{T}\right)} \leq T^{(q-n) / 2 q} C_{10}^{*} .
$$

Combining (2.22), (2.23), (2.17) and (2.29)-(2.30), we obtain

$$
\left\|\left(\frac{\partial v^{k}}{\partial \nu}-\frac{\partial v_{0}}{\partial \nu}\right) u^{(k)}\right\|_{W_{q}^{1-1 / q, 1 / 2-1 / 2 q}\left(\Gamma_{T}\right)} \leq T^{(q-n) / 2 q} C_{11}^{*} .
$$

From $(2.15),(2.21)$ and $(2.31)$ we arrive at the following inequality:

$$
\left\|u^{(k+1)}\right\|_{W_{q}^{2,1}\left(Q_{T}\right)} \leq C_{0}^{*}(T)\left(\left\|u_{0}\right\|_{W^{2, q}(\Omega)}+T^{(q-n) / 2 q} C_{12}^{*}\right) .
$$

We fix $0<\delta \ll 1$. Noting that $C_{0}^{*}$ and $C_{12}^{*}$ are monotone increasing on $\mathrm{T}$, we can take $M$ and $T$ in (2.13) such that

$$
M=2\left\|u_{0}\right\|_{W^{2, q}(\Omega)} \max \left\{1, C_{0}^{*}(\delta)\right\}, \quad T^{(q-n) / 2 q} C_{12}^{*}(\delta) \leq\left\|u_{0}\right\|_{W^{2, q}(\Omega)} .
$$


Then we can define a mapping

$$
\Psi: X_{M, T} \ni u^{(k)} \mapsto u^{(k+1)} \in X_{M, T} .
$$

To prove the existence of the solution in local-time, it suffices to prove that $\Psi$ is a contraction mapping on $X_{M, T}$. But we omit the proof since the calculations are similar to the above ones which assure that $\Psi$ is a mapping on $X_{M, T}$. We may need to replace $T$ with $\tilde{T}$ which is sufficiently smaller than the previous $T$. Fix $M>0$ and $0<T \ll 1$ such that $\Psi$ is a contraction mapping on $X_{M, T}$, and let $(u, v)$ be a solution to (GCZ) satisfying $u \in X_{M, T}$. The uniqueness of the solution is reduced to that of $u$ by Lemma 4 . Let $\left(u_{1}, v_{1}\right)$ be another solution to (GCZ). By the above argument, we see that there exist $M_{1}$ and $T_{1}$ such that $\Psi$ is a contraction mapping on $X_{M_{1}, T_{1}}$. If we set

$$
M^{\prime}:=\max \left\{M, M_{1}\right\}, \quad T^{\prime}:=\min \left\{T, T_{1}\right\},
$$

then both $u$ and $u_{1}$ are in $X_{M^{\prime}, T^{\prime}}$. Since $\Psi$ is also a contraction mapping on $X_{M^{\prime}, T^{\prime}}$, the fixed point is unique in $X_{M^{\prime}, T^{\prime}}$, and therefore

$$
u_{1}=u_{2} \quad \text { in } Q_{T^{\prime}} .
$$

The uniqueness, $u=u_{1}$ in $Q_{T}$, is clear if $T^{\prime}=T$. Otherwise, we take

$$
T_{0}:=\inf \left\{\tau \in(0, T) \mid u \neq u_{1} \text { in } Q_{\tau}\right\} \in\left[T_{1}, T\right) .
$$

It holds that $\sup _{t \in\left[0, T_{0}\right)}\left\|u_{1}(t)\right\|_{W_{q}^{2,1}\left(Q_{T}\right)} \leq C$ for some $C>0$. In fact, smoothness of the solution in $\overline{Q_{T_{0}}}$ follows from (2.11), (2.12), (2.14), Lemma 4 and the Schauder theory with (1.9), see [14]. Therefore, there exists $0<\delta \ll 1$ such that $u=u_{1}$ in $Q_{T_{0}+\delta}$, which is a contradiction by the definition of $T_{0}$. Thus the uniqueness is shown. Smoothness of the solution is similar to the above. The proof of Theorem 1 is complete.

\section{Proof of Theorem 2}

We confirm several facts used later (see [21], [23]). The Gagliardo-Nirenberg inequality in two space dimentions is described by

$$
\|w\|_{2}^{2} \leq K^{2}\left(\|\nabla w\|_{1}^{2}+\|w\|_{1}^{2}\right) \quad \text { for all } w \in W^{1,1}(\Omega),
$$

where $K$ is a constant determined by $\Omega$. We put $B_{R}\left(x_{0}\right)=\left\{x \in \boldsymbol{R}^{2}|| x-x_{0} \mid<\right.$ $R\}$. To derive local in space estimates, we take the smooth cut-off function $\varphi$ satisfying

$$
0 \leq \varphi \leq 1 \quad \text { in } \boldsymbol{R}^{2}, \quad \frac{\partial \varphi}{\partial \nu}=0 \quad \text { on } \partial \Omega .
$$


First, given $x_{0} \in \Omega$ and $0<R^{\prime}<R$ with $B_{2 R}\left(x_{0}\right) \subset \Omega$, we take such $\varphi$ by

$$
\varphi(x)= \begin{cases}1 & \left(x \in B_{R^{\prime}}\left(x_{0}\right)\right) \\ 0 & \left(x \in \boldsymbol{R}^{2} \backslash B_{R}\left(x_{0}\right)\right) .\end{cases}
$$

Given $x_{0} \in \partial \Omega$, next, we take a smooth conformal mapping $X: B_{2 R} \cap \bar{\Omega} \rightarrow \boldsymbol{R}^{2}$ satisfying $x_{0} \mapsto 0$ and

$$
\begin{aligned}
& X\left(B_{2 R}\left(x_{0}\right) \cap \Omega\right) \subset\left\{\left(x_{1}, x_{2}\right) \mid x_{2}>0\right\} \\
& X\left(B_{2 R}\left(x_{0}\right) \cap \partial \Omega\right) \subset\left\{\left(x_{1}, x_{2}\right) \mid x_{2}=0\right\}, \\
& X\left(B_{R^{\prime}}\left(x_{0}\right) \cap \Omega\right) \subset B_{1 / 2}(0), \quad X\left(\Omega \backslash B_{R}\left(x_{0}\right)\right) \subset \boldsymbol{R}^{2} \backslash B_{1}(0)
\end{aligned}
$$

for $0<R^{\prime}<R \ll 1$. Then set $\varphi(x):=\eta(X(x))$, where $\eta \in C_{0}^{\infty}\left(\boldsymbol{R}^{2}\right), 0 \leq \eta \leq 1$, $\eta=1$ in $B_{1 / 2}(0)$, and $\eta=0$ in $\boldsymbol{R}^{2} \backslash B_{1}(0)$. It holds that

$$
\frac{\partial}{\partial \nu}(\eta \circ X)=\frac{\partial X}{\partial \nu} \cdot(\nabla \eta \circ X)=0 \quad \text { on } \partial \Omega
$$

because $(\partial X) /(\partial \nu)$ is proportional to $(0,1)$.

The above $\varphi$ is sometimes written as $\varphi_{x_{0}, R^{\prime}, R}$. Then, $\psi:=\left(\varphi_{x_{0}, R^{\prime}, R}\right)^{6}$ satisfies

$$
\begin{aligned}
& \psi(x)= \begin{cases}1 & \left(x \in B_{R^{\prime}}\left(x_{0}\right)\right) \\
0 & \left(x \in \boldsymbol{R}^{2} \backslash B_{R}\left(x_{0}\right)\right),\end{cases} \\
& 0 \leq \psi \leq 1 \quad \text { in } \boldsymbol{R}^{2}, \quad \frac{\partial \psi}{\partial \nu}=0 \quad \text { on } \partial \Omega, \\
& |\nabla \psi| \leq A \psi^{5 / 6}, \quad|\Delta \psi| \leq B \psi^{2 / 3} \quad \text { in } \boldsymbol{R}^{2},
\end{aligned}
$$

where $\mathrm{A}$ and $\mathrm{B}$ are positive constants determined by $R^{\prime}$ and $R$. We use the following estimates derived from $(3.1)$, see $[21,23]$ for the proof.

Lemma 5. The following inequalities hold for any $s>1$, where $C>0$ is a constant:

$$
\begin{aligned}
& \int_{\Omega} u^{2} \psi d x \leq 2 K^{2} \int_{B_{R}\left(x_{0}\right) \cap \Omega} u d x \cdot \int_{\Omega} u^{-1}|\nabla u|^{2} \psi d x+K^{2}\left(\frac{A^{2}}{2}+1\right)\|u\|_{1}^{2} \\
& \int_{\Omega} u^{2} d x \leq \frac{2 K^{2}}{\log s} \int_{\Omega}\left(u \log u+e^{-1}\right) d x \cdot \int_{\Omega} u^{-1}|\nabla u|^{2} d x+2 K^{2}\|u\|_{1}^{2}+3 s^{2}|\Omega|, \\
& \int_{\Omega} u^{3} \psi d x \leq \frac{72 K^{2}}{\log s} \int_{B_{R}\left(x_{0}\right) \cap \Omega}\left(u \log u+e^{-1}\right) d x \cdot \int_{\Omega}|\nabla u|^{2} \psi d x \\
&+C\|u\|_{L^{1}\left(B_{R}\left(x_{0}\right) \cap \Omega\right)}^{3}+10|\Omega| s^{3} .
\end{aligned}
$$


Lemma 5 is irrelevant to (GCZ) and the above $u$ is not necessarily to be the solution. Here, we emphasize the mass conservation, i.e.

$$
\|u(t)\|_{1}=\left\|u_{0}\right\|_{1}=\mathrm{constant} \quad \text { for } 0 \leq t<T_{\max } .
$$

This property is derived from the first boundary condition of (GCZ) and the non-negativity of $u$. The second equation of (GCZ) is written as

$$
-\Delta v+v=-f(v)+u \quad \text { in } \Omega, \quad \frac{\partial v}{\partial \nu}=-g(v) \quad \text { on } \partial \Omega .
$$

Since $|f(v)|=-f(v) \leq-f(0)=|f(0)|$ if $f(v) \leq 0$ by (1.4)-(1.5), we obtain

$$
|f(v)| \leq|f(0)|+f(v)
$$

and hence

$$
\begin{aligned}
\int_{\Omega}|f(v)| d x & \leq|\Omega| \cdot|f(0)|+\int_{\Omega} f(v) d x \\
& =|\Omega| \cdot|f(0)|-\int_{\partial \Omega} g(v) d S-\int_{\Omega} v d x+\int_{\Omega} u d x \\
& \leq|\Omega| \cdot|f(0)|+|\partial \Omega| \cdot|g(0)|+\|u\|_{1} \leq \text { constant. }
\end{aligned}
$$

It also holds that

$$
\begin{aligned}
\int_{\partial \Omega}|g(v)| d S & \leq|\partial \Omega| \cdot|g(0)|+\int_{\partial \Omega} g(v) d S \\
& =|\partial \Omega| \cdot|g(0)|+\int_{\Omega}-v-f(v)+u d x \\
& \leq|\partial \Omega| \cdot|g(0)|+\int_{\Omega}|f(v)| d x+\|u\|_{1} \leq \text { constant. }
\end{aligned}
$$

Then, we apply the $L^{1}$ estimate of [7] to obtain

$$
\begin{array}{rlr}
\sup _{0 \leq t<T_{\max }}\|v(t)\|_{W^{1, q}} \leq C_{q} & \text { for } q \in[1,2), \\
\sup _{0 \leq t<T_{\max }\|v(t)\|_{q}} \leq C_{q} & \text { for } q \in[1, \infty), \\
\sup _{0 \leq t<T_{\max }}\|v(t)\|_{L^{q}(\partial \Omega)} \leq C_{q} & \text { for } q \in[1, \infty),
\end{array}
$$

by Sobolev's and the trace embedding theorems, see [1].

Henceforth, we assume $T_{\max }<\infty$. 
Lemma 6. We obtain $x_{0} \in \mathcal{B}$ if and only if

$$
\limsup _{t \uparrow T_{\max }} \int_{B_{R}\left(x_{0}\right) \cap \Omega} u \log u d x=\infty
$$

for any $0<R \ll 1$.

Proof. The 'if' part is obvious by the definition of $\mathcal{B}$. To show the converse, we assume

$$
\limsup _{t \uparrow T_{\max }} \int_{B_{R}\left(x_{0}\right) \cap \Omega} u \log u d x<\infty
$$

for some $0<R \ll 1$ and show $x_{0} \notin \mathcal{B}$. In fact, this implies

$$
\underset{t \uparrow T_{\max }}{\limsup } \int_{\Omega}(u \log u) \psi d x<\infty
$$

for $\psi=\left(\varphi_{x_{0}, R^{\prime}, R}\right)^{6}\left(0<R^{\prime}<R\right)$. Multiplying the fist equation of (GCZ) by $u \psi$ and integrating over $\Omega$, we have

$$
\begin{aligned}
& \frac{1}{2} \frac{d}{d t} \int_{\Omega} u^{2} \psi d x+\int_{\Omega}|\nabla u|^{2} d x+\int_{\Omega} u \nabla u \cdot \nabla \psi d x \\
= & \int_{\Omega} u \psi \nabla u \cdot \nabla v d x+\int_{\Omega} u^{2} \nabla v \cdot \nabla \psi d x .
\end{aligned}
$$

The first term of the right hand of (3.8) is equal to

$$
\begin{aligned}
\int_{\Omega} u \psi \nabla u \cdot \nabla v d x & =\frac{1}{2} \int_{\Omega} \psi \nabla u^{2} \cdot \nabla v d x \\
& =I-\frac{1}{2} \int_{\Omega} u^{2} \psi \Delta v d x-\frac{1}{2} \int_{\Omega} u^{2} \nabla v \cdot \nabla \psi d x
\end{aligned}
$$

where

$$
I:=-\frac{1}{2} \int_{\partial \Omega} \psi u^{2} g(v) d S .
$$

Then, using the second equation of (GCZ) and (1.4)-(1.5), we obtain

$$
\begin{aligned}
& \int_{\Omega} u \psi \nabla u \cdot \nabla v d x \\
= & I-\frac{1}{2} \int_{\Omega} u^{2}(v+f(v)) \psi d x+\frac{1}{2} \int_{\Omega} u^{3} \psi d x-\frac{1}{2} \int_{\Omega} u^{2} \nabla v \cdot \nabla \psi d x \\
\leq & I+\frac{1}{2}|f(0)| \int_{\Omega} u^{2} \psi d x+\frac{1}{2} \int_{\Omega} u^{3} \psi d x-\frac{1}{2} \int_{\Omega} u^{2} \nabla v \cdot \nabla \psi d x
\end{aligned}
$$




$$
\begin{aligned}
\leq I+\frac{1}{2}|f(0)| \int_{\Omega} u^{2} \psi d x+\frac{1}{2} \int_{\Omega} u^{3} \psi d x & \\
& +\frac{1}{2} \int_{\Omega} v \nabla u^{2} \cdot \nabla \psi d x+\frac{1}{2} \int_{\Omega} u^{2} v \Delta \psi d x .
\end{aligned}
$$

From (3.8)-(3.11) it follows that

$$
\begin{aligned}
& \frac{1}{2} \frac{d}{d t} \int_{\Omega} u^{2} \psi d x+\int_{\Omega}|\nabla u|^{2} \psi d x+\int_{\Omega} u \nabla u \cdot \nabla \psi d x \\
\leq & I+\frac{1}{2}|f(0)| \int_{\Omega} u^{2} \psi d x+\frac{1}{2} \int_{\Omega} u^{3} \psi d x \\
& -\frac{1}{2} \int_{\Omega} v \nabla u^{2} \cdot \nabla \psi d x-\frac{1}{2} \int_{\Omega} u^{2} v \Delta \psi d x .
\end{aligned}
$$

Using Young's inequality, we get the following inequalities:

$$
\begin{aligned}
& \frac{1}{2}\left|\int_{\Omega} u^{2} v \Delta \psi d x\right| \leq \frac{1}{2} \int_{\Omega} u^{2} \psi^{2 / 3} \cdot B v d x \\
& \leq \frac{1}{3} \int_{\Omega} u^{3} \psi d x+\frac{B^{3}}{6}\|v\|_{3}^{3}, \\
& \frac{1}{2}\left|\int_{\Omega} v \nabla u^{2} \cdot \nabla \psi d x\right| \leq \int_{\Omega}|\nabla u| \psi^{1 / 2} \cdot u \psi^{1 / 3} \cdot A v d x \\
& \leq \frac{1}{4} \int_{\Omega}|\nabla u|^{2} \psi d x+\int_{\Omega} u^{2} \psi^{2 / 3} \cdot A^{2} v^{2} d x \\
& \leq \frac{1}{4} \int_{\Omega}|\nabla u|^{2} \psi d x+\frac{1}{3} \int_{\Omega} u^{3} \psi d x+\frac{4 A^{6}}{3}\|v\|_{6}^{6},
\end{aligned}
$$

and

$$
\begin{aligned}
\left|\int_{\Omega} u \nabla u \cdot \nabla \psi d x\right| & \leq \int_{\Omega}|\nabla u| \psi^{1 / 2} \cdot u \psi^{1 / 3} \cdot A d x \\
& \leq \frac{1}{4} \int_{\Omega}|\nabla u|^{2} \psi d x+\frac{1}{3} \int_{\Omega} u^{3} \psi d x+\frac{4 A^{6}}{3}|\Omega| .
\end{aligned}
$$

Combining (3.12)-(3.15), we obtain

$$
\frac{1}{2} \frac{d}{d t} \int_{\Omega} u^{2} \psi d x+\frac{1}{2} \int_{\Omega}|\nabla u|^{2} \psi d x \leq I+\frac{1}{2}|f(0)| \int_{\Omega} u^{2} \psi d x+\frac{3}{2} \int_{\Omega} u^{3} \psi d x+C_{1},
$$

where $C_{1}>0$ is an absolute constant induced by (3.4). 
Now, we estimate $I$ defined by (3.10). We see that there exists an absolute constant $M_{1}$ (only depending on $g(0)$ ) such that

$$
I \leq M_{1} \int_{\partial \Omega} u^{2} \psi d S
$$

by (1.3)-(1.5). We take a constant $L$ such that

$$
\|w\|_{L^{1}(\partial \Omega)} \leq M_{2}\|w\|_{W^{1,1}(\Omega)} \quad \text { for all } w \in W^{1,1}(\Omega) .
$$

Fix $0<\varepsilon_{i} \ll 1(i=1,2)$. It holds that

$$
\begin{aligned}
\left\|u^{2} \psi\right\|_{W^{1,1}} & =\int_{\Omega} u^{2} \psi d x+\int_{\Omega}\left|\nabla\left(u^{2} \psi\right)\right| d x \\
\int_{\Omega}\left|\nabla\left(u^{2} \psi\right)\right| d x & \leq 2 \int_{\Omega} u|\nabla u| \psi d x+\int_{\Omega} u^{2}|\nabla \psi| d x \\
2 \int_{\Omega} u|\nabla u| \psi d x & =2 \int_{\Omega} u \psi^{1 / 2} \cdot|\nabla u| \psi^{1 / 2} d x \\
& \leq 2 \varepsilon_{1} \int_{\Omega}|\nabla u|^{2} \psi d x+2 \varepsilon_{1}^{-1} \int_{\Omega} u^{2} \psi d x
\end{aligned}
$$

and

$$
\int_{\Omega} u^{2}|\nabla \psi| d x \leq \int_{\Omega} u^{2} \psi^{2 / 3} \cdot A \psi^{1 / 6} d x \leq \varepsilon_{2} \int_{\Omega} u^{3} \psi d x+\frac{4}{27} \varepsilon_{2}^{-2} A^{6}|\Omega| .
$$

Combining (3.17)-(3.22), we get

$$
\begin{aligned}
I \leq & M_{1} M_{2}\left\{\left(1+2 \varepsilon_{1}^{-1}\right) \int_{\Omega} u^{2} \psi d x+2 \varepsilon_{1} \int_{\Omega}|\nabla u|^{2} \psi d x\right. \\
& \left.+\varepsilon_{2} \int_{\Omega} u^{3} \psi d x+\frac{4}{27} \varepsilon_{2}^{-2} A^{6}|\Omega|\right\} .
\end{aligned}
$$

From (3.12) and (3.23), we have

$$
\begin{aligned}
& \frac{1}{2} \frac{d}{d t} \int_{\Omega} u^{2} \psi d x+\left(\frac{1}{2}-2 \varepsilon_{1} M_{1} M_{2}\right) \int_{\Omega}|\nabla u|^{2} \psi d x \\
& \leq C_{1}+\frac{4}{27} \varepsilon_{2}^{-2} M_{1} M_{2} A^{6}|\Omega|+\left(\frac{3}{2}+\varepsilon_{2} M_{1} M_{2}\right) \int_{\Omega} u^{3} \psi d x \\
&+\left\{\left(1+2 \varepsilon_{1}^{-1}\right) M_{1} M_{2}+\frac{1}{2}|f(0)|\right\} \int_{\Omega} u^{2} \psi d x .
\end{aligned}
$$


Taking $\varepsilon_{1}=\left(8 M_{1} M_{2}\right)^{-1}, \varepsilon_{2}=\left(2 M_{1} M_{2}\right)^{-1}$, we have

$$
\begin{aligned}
& \frac{d}{d t} \int_{\Omega} u^{2} \psi d x+\frac{1}{2} \int_{\Omega}|\nabla u|^{2} d x \\
\leq & C_{2}+4 \int_{\Omega} u^{3} \psi d x+\left\{2\left(1+16 M_{1} M_{2}\right)+|f(0)|\right\} \int_{\Omega} u^{2} \psi d x .
\end{aligned}
$$

Now we use (3.7) and prescribe $s \gg 1$ by

$$
\frac{72 K^{2}}{\log s} \int_{B_{R}\left(x_{0}\right) \cap \Omega}\left(u \log u+e^{-1}\right) d x \leq \frac{1}{8} .
$$

Then it follows that

$$
\frac{d}{d t} \int_{\Omega} u^{2} \psi d x \leq C_{3}+\left\{2\left(1+16 M_{1} M_{2}\right)+|f(0)|\right\} \int_{\Omega} u^{2} \psi d x
$$

by Lemma 5 . We use Gronwall's inequality and our assumption $T=T_{\max }<\infty$ to get

$$
\sup _{0 \leq t<T} \int_{\Omega} u^{2} \psi d x<\infty .
$$

We continue the process, multiplying the first equation of (GCZ) by $u^{2} \psi$ and integrating by parts. We have

$$
\begin{aligned}
& \frac{1}{3} \frac{d}{d t} \int_{\Omega} u^{3} \psi+2 \int_{\Omega} u|\nabla u|^{2}+\int_{\Omega} u^{2} \nabla u \cdot \nabla \psi d x \\
& =2 \int_{\Omega} u^{2}(\nabla u \cdot \nabla v) \psi d x+\int_{\Omega} u^{3} \nabla v \cdot \nabla \psi d x,
\end{aligned}
$$

which is equivalent to

$$
\begin{aligned}
& \frac{d}{d t} \int_{\Omega} w^{2} \psi d x+\frac{8}{3} \int_{\Omega}|\nabla w|^{2} \psi d x+2 \int_{\Omega} w \nabla w \cdot \nabla \psi d x \\
= & 4 \int_{\Omega} w(\nabla w \cdot \nabla v) \psi d x+3 \int_{\Omega} w^{2} \nabla v \cdot \nabla \psi d x,
\end{aligned}
$$

where $w:=u^{3 / 2}$. Using the second equation of (GCZ), we have

$$
\begin{aligned}
& 4 \int_{\Omega} w(\nabla w \cdot \nabla v) \psi d x=2 \int_{\Omega}\left(\nabla w^{2} \cdot \nabla v\right) \psi d x \\
= & I I-2 \int_{\Omega} w^{2} \psi \Delta v d x-2 \int_{\Omega} w^{2} \nabla v \cdot \nabla \psi d x
\end{aligned}
$$




$$
\begin{aligned}
& =I I-2 \int_{\Omega} w^{2}(v+f(v)) \psi d x+2 \int_{\Omega} w^{8 / 3} \psi d x-2 \int_{\Omega} w^{2} \nabla v \cdot \nabla \psi d x \\
& \leq I I+2|f(0)|+2 \int_{\Omega} w^{2} \psi d x+2 \int_{\Omega} w^{8 / 3} \psi d x-2 \int_{\Omega} w^{2} \nabla v \cdot \nabla \psi d x,
\end{aligned}
$$

where

$$
I I:=-2 \int_{\partial \Omega} \psi w^{2} g(v) d S .
$$

Then, it follows that

$$
\begin{aligned}
& \frac{d}{d t} \int_{\Omega} w^{2} \psi d x+\frac{8}{3}|\nabla w|^{2} \psi d x+2 \int_{\Omega} w \nabla w \cdot \nabla \psi d x \\
\leq \quad I I+2|f(0)| & \int_{\Omega} w^{2} \psi d x+2 \int_{\Omega} w^{8 / 3} \psi d x \\
& -\int_{\Omega} v \nabla w^{2} \cdot \nabla \psi d x-\int_{\Omega} v w^{2} \Delta \psi d x .
\end{aligned}
$$

By the calculation analogous to (3.13)-(3.15) and (3.23), we get the folowing inequalities:

$$
\begin{aligned}
2\left|\int_{\Omega} w \nabla w \cdot \nabla \psi d x\right| & \leq \frac{1}{3} \int_{\Omega}|\nabla w|^{2} \psi d x+3 \int_{\Omega} w^{3} \psi d x+\frac{4}{9} A^{6}|\Omega| \\
& 2 \int_{\Omega} w^{8 / 3} \psi d x \leq 2 \int_{\Omega} w^{3} \psi d x+\left(\frac{8}{9}\right)^{8} \frac{2}{9}|\Omega| \\
\left|\int_{\Omega} v \nabla w^{2} \cdot \nabla \psi d x\right| \leq & \frac{1}{3} \int_{\Omega}|\nabla w|^{2} \psi d x+3 \int_{\Omega} w^{3} \psi d x+\frac{4}{9} A^{6}\|v\|_{6}^{6} \\
& \left|\int_{\Omega} v w^{2} \Delta \psi d x\right| \leq \int_{\Omega} w^{3} \psi d x+\frac{4}{27} B^{3}\|v\|_{3}^{3} \\
I I \leq 4 M_{1} M_{2}\left\{\left(1+2 \varepsilon_{1}^{-1}\right) \int_{\Omega} u^{2} \psi d x+2 \varepsilon_{1} \int_{\Omega}|\nabla u|^{2} \psi d x\right. & \left.+\varepsilon_{2} \int_{\Omega} u^{3} \psi d x+\frac{4}{27} \varepsilon_{2}^{-2} A^{6}|\Omega|\right\}
\end{aligned}
$$

where $\varepsilon_{1}$ and $\varepsilon_{2}$ are arbitrary positive constants. Inequalities (3.27)-(3.30) are summarized by

$$
\begin{aligned}
& \frac{d}{d t} \int_{\Omega} w^{2} \psi d x+\left(2-8 \varepsilon_{1} M_{1} M_{2}\right) \int_{\Omega}|\nabla w|^{2} \psi d x \\
\leq & C_{4}+\frac{16}{27} \varepsilon_{2}^{-2} M_{1} M_{2} A^{6}|\Omega|+\left(9+4 \varepsilon_{2} M_{1} M_{2}\right) \int_{\Omega} w^{3} \psi d x
\end{aligned}
$$




$$
+\left\{4 M_{1} M_{2}\left(1+2 \varepsilon_{1}^{-1}\right)+2|f(0)|\right\} \int_{\Omega} w^{2} \psi d x,
$$

where $C_{4}>0$ is an absolute constant induced by (3.4). If we take $\varepsilon_{1}=$ $\left(16 M_{1} M_{2}\right)^{-1}$ and $\varepsilon_{2}=\left(4 M_{1} M_{2}\right)^{-1}$, then it holds that

$$
\begin{aligned}
\frac{d}{d t} \int_{\Omega} w^{2} \psi d x & +\frac{3}{2} \int_{\Omega}|\nabla w|^{2} \psi d x \leq C_{5}+10 \int_{\Omega} w^{3} \psi d x \\
& +\left\{4 M_{1} M_{2}\left(1+32 M_{1} M_{2}\right)+2|f(0)|\right\} \int_{\Omega} w^{2} \psi d x
\end{aligned}
$$

Since (3.25) implies

$$
\begin{array}{r}
\sup _{0 \leq t<T} \int_{B_{R^{\prime}}\left(x_{0}\right) \cap \Omega} w \log w d x<\infty \\
\sup _{0 \leq t<T}\|w\|_{L^{1}\left(B_{R^{\prime}}\left(x_{0}\right) \cap \Omega\right)}<\infty
\end{array}
$$

for $R^{\prime} \in(0, R)$, we can repeat the argument of the previous stage. We see

$$
\sup _{0 \leq t<T}\|w\|_{L^{2}\left(B_{r}\left(x_{0}\right) \cap \Omega\right)}^{2 / 3}=\sup _{0 \leq t<T}\|u\|_{L^{3}\left(B_{r}\left(x_{0}\right) \cap \Omega\right)}<\infty,
$$

replacing $u, R$ and $\psi=\left(\varphi_{x_{0}, R^{\prime}, R}\right)^{6}$ by $w, R^{\prime}$, and $\psi=\left(\varphi_{x_{0}, R^{\prime \prime}, R^{\prime}}\right)^{6}$ respectively, where $R^{\prime \prime} \in\left(0, R^{\prime}\right)$ and $r \in(0, R)$. For $h:=u-f(v)$, we have

$$
\sup _{0 \leq t<T}\|h\|_{L^{3}\left(B_{r}\left(x_{0}\right) \cap \Omega\right)}<\infty
$$

by (3.4) and (3.32), and then

$$
\sup _{0 \leq t<T}\|v\|_{W^{2,3}\left(B_{r^{\prime}}\left(x_{0}\right) \cap \Omega\right)}<\infty \quad \text { for } r^{\prime} \in(0, r),
$$

follows from the elliptic regularity. Since $R^{\prime} \in(0, R)$ and $r^{\prime} \in\left(0, R^{\prime}\right)$ are arbitrary, Sobolev's embedding guarantees

$$
\sup _{0 \leq t<T}\|v\|_{C^{1}\left(B_{r}\left(x_{0}\right) \cap \Omega\right)}<\infty \quad \text { for all } r \in(0, R) .
$$

Repeating the arguments once more, we have

$$
\sup _{0 \leq t<T}\|u\|_{L^{4}\left(B_{r}\left(x_{0}\right) \cap \Omega\right)}<\infty \quad \text { for all } r \in(0, R) .
$$

From this stage, we use only the first equation of (GCZ). The rest of the proof is thus anagolous to Step 3 of Lemma 5 of [21] (see also [23]). Skipping 
details, we just mention that estimates (3.33)-(3.34) and the Moser's iteration scheme (see [3]) are used to obtain

$$
\sup _{0 \leq t<T}\|u \psi\|_{\infty}<\infty .
$$

This implies

$$
\limsup _{t \uparrow T}\|u\|_{L^{\infty}\left(B_{r^{\prime}}\left(x_{0}\right) \cap \Omega\right)}<\infty,
$$

and therefore $x_{0} \notin \mathcal{B}$.

Proof of Theorem 2. We shall prove that

$$
\limsup _{t \uparrow T} \int_{\Omega} u \log u d x<\infty
$$

follows from

$$
\liminf _{t \uparrow T} \int_{\Omega} u \log u d x<\infty .
$$

In fact, the proof of Lemma 6 is valid even to $\varphi \equiv 1$, and hence we can show that (3.35) implies

$$
\limsup _{t \uparrow T}\|u\|_{\infty}<\infty .
$$

If (3.37) is the case, then the standard theories (see [2], [12] and [14]) and (3.3)(3.5) guarantee that the solution $u$ to (GCZ) is continued after $t=T$. Thus, $T_{\max }<\infty$ implies (3.36) and hence we obtain the result $\lim _{t \uparrow T} \int_{\Omega} u \log u d x=$ $\infty$.

Now, multiplying $\log u$ by the first equation of (GCZ), and we have

$$
\begin{aligned}
\frac{d}{d t} \int_{\Omega} u \log u d x+\int_{\Omega} u^{-1}|\nabla u|^{2} & +u v+u(f(v)-f(0)) d x \\
& =|f(0)| \cdot\|u\|_{1}+\int_{\Omega} u^{2} d x .
\end{aligned}
$$

Using Lemma 5 and (3.2), we see

$$
\begin{array}{r}
\frac{d}{d t} \int_{\Omega} u \log u d x+\left\{1-\frac{2 K^{2}}{\log s} \int_{\Omega}\left(u \log u+e^{-1}\right) d x\right\} \int_{\Omega} u^{-1}|\nabla u|^{2} d x \\
\leq C_{6}\left\|u_{0}\right\|_{1}^{2}+3 s^{2}|\Omega|,
\end{array}
$$

where $s>0$ is arbitrary. Then, we take

$$
s=s(t)=\exp \left(2 K^{2} \int_{\Omega}\left(u \log u+e^{-1}\right) d x\right)>1,
$$


and obtain

$$
\frac{d J}{d t} \leq C_{6}\left\|u_{0}\right\|_{1}^{2}+3|\Omega| \exp \left(4 K^{2} J\right), \quad J(t):=\int_{\Omega}\left(u \log u+e^{-1}\right) d x .
$$

From this differential inequality, we can conclude that $(3.36) \Rightarrow(3.35)$.

\section{Proof of Theorem 3}

We take the Green's function $G=G\left(x, x^{\prime}\right)$ defined by

$$
\left(-\Delta_{x^{\prime}}+1\right) G=\delta\left(x^{\prime}-x\right)\left(x^{\prime} \in \Omega\right), \quad \frac{\partial}{\partial \nu_{x^{\prime}}} G=0\left(x^{\prime} \in \partial \Omega\right)
$$

for $x \in \Omega$, where $\delta$ denotes the Dirac' delta function, and shall use the following lemma (see [21],[23] for the proof):

Lemma 7. It holds that $\rho \in L^{\infty}(\Omega \times \Omega)$, where

$$
\rho\left(x, x^{\prime}\right)=\nabla \psi(x) \cdot \nabla_{x} G\left(x, x^{\prime}\right)+\nabla \psi\left(x^{\prime}\right) \cdot \nabla_{x^{\prime}} G\left(x, x^{\prime}\right) .
$$

First, we show the following lemma:

Lemma 8. It holds that

$$
\frac{d}{d t} \int_{\Omega}(u \log u) \psi+\frac{1}{4} u^{-1}|\nabla u|^{2} \psi d x \leq 5 \int_{\Omega} u^{2} \psi d x+C_{8} .
$$

Proof. Multiplying $\psi \log u$ to the first equation of (GCZ), we have

$$
\begin{aligned}
\frac{d}{d t} \int_{\Omega}(u \log u) \psi d x= & -\int_{\Omega} \nabla u \cdot \nabla\{(\log u+1) \psi\} d x \\
& +\int_{\Omega} u \nabla v \cdot \nabla\left\{(\log u+1) \psi d x=:-F_{1}+F_{2} .\right.
\end{aligned}
$$

From the second equation of (GCZ)

$$
\begin{aligned}
F_{2} & =\int_{\Omega} \nabla v \cdot \nabla u d x+\int_{\Omega} u(\log u+1) \nabla v \cdot \nabla \psi d x \\
& =I I I-\int_{\Omega} u \nabla \cdot(\psi \nabla v) d x+\int_{\Omega} u(\log u+1) \nabla v \cdot \nabla \psi d x
\end{aligned}
$$




$$
=I I I+\int_{\Omega} u^{2} \psi d x-\int_{\Omega} u \psi(v+f(v)) d x+\int_{\Omega}(u \log u) \nabla v \cdot \nabla \psi d x,
$$

where

$$
I I I:=\int_{\partial \Omega} u \psi g(v) d S
$$

We also have that

$$
F_{1}=\int_{\Omega} u^{-1}|\nabla u|^{2} \psi d x+\int_{\Omega}(\log u+1) \nabla u \cdot \nabla \psi d x .
$$

Then we obtain the following inequality:

$$
\begin{aligned}
& \frac{d}{d t} \int_{\Omega}(u \log u) \psi d x+\int_{\Omega} u^{-1}|\nabla u|^{2} \psi d x+\int_{\Omega} u \psi(v+f(v)-f(0)) d x \\
= & I I I+|f(0)| \int_{\Omega} u \psi d x-\int_{\Omega}(\log u+1) \nabla u \cdot \nabla \psi d x+\int_{\Omega}(u \log u) \nabla v \cdot \nabla \psi d x .
\end{aligned}
$$

Here, we recall the elementary inequality: Let $\alpha>0$ and $0<\beta<2$. Then it holds that

$$
(|\log u|+1)^{\alpha} u^{\beta} \leq u^{2}+d_{\alpha, \beta},
$$

where $k_{\alpha, \beta}$ is a positive constant determined by only $\alpha$ and $\beta$.

$$
\begin{aligned}
& \left|\int_{\Omega}(\log u+1) \nabla u \cdot \nabla \psi d x\right| \leq A \int_{\Omega}(|\log u|+1) u^{1 / 2} \psi^{1 / 3} \cdot u^{-1 / 2}|\nabla u| \psi^{1 / 2} d x \\
& \leq A|\partial \Omega|^{1 / 6}\left\{\int_{\Omega}(|\log u|+1)^{3} u^{3 / 2} \psi d x\right\}^{1 / 3}\left\{\int_{\Omega} u^{-1}|\nabla u|^{2} \psi d x\right\}^{1 / 2} \\
& \leq A|\partial \Omega|^{1 / 6}\left\{\int_{\Omega} u^{2} \psi d x+d_{3,3 / 2}|\Omega|\right\}^{1 / 3}\left\{\int_{\Omega} u^{-1}|\nabla u|^{2} \psi d x\right\}^{1 / 2} \\
& \leq \int_{\Omega} \frac{1}{4} u^{-1}|\nabla u|^{2} \psi u^{2} \psi d x+d_{3,3 / 2}|\Omega|+\frac{4}{27} A^{6}|\Omega| .
\end{aligned}
$$

The fifth term of the right-hand side of equality (4.2) is equal to

$$
-\int_{\Omega} v \nabla \cdot(u \log u \nabla \psi) d x=-\int_{\Omega} v(\log u+1) \nabla u \cdot \nabla \psi d x-\int_{\Omega}(v u \log u) \Delta \psi d x
$$

by $\left.\frac{\partial \psi}{\partial \nu}\right|_{\partial \Omega}=0$. Each term of the right-hand side of equality (4.4) is estimated as follows:

$$
\left|\int_{\Omega} v(\log u+1) \nabla u \cdot \nabla \psi d x\right|
$$




$$
\begin{aligned}
& \leq A \int_{\Omega} v \cdot u^{1 / 2}(|\log u|+1) \psi^{1 / 3} \cdot u^{-1 / 2}|\nabla u| \psi^{1 / 2} d x \\
& \leq A\|v\|_{6}\left\{u^{3 / 2}(|\log u|+1)^{3} \psi d x\right\}^{1 / 3}\left\{u^{-1}|\nabla u|^{2} \psi d x\right\}^{1 / 2} \\
& \leq \frac{1}{4} \int_{\Omega} u^{2} \psi d x+d_{3,3 / 2}|\Omega|+\frac{4}{27} A^{6}\|v\|_{6}^{6} . \\
& \quad\left|\int_{\Omega}(v u \log u) \Delta \psi d x\right| \leq B \int_{\Omega} v|u \log u| \psi^{2 / 3} d x \\
& \leq B\|v\|_{3}\left\{\int_{\Omega} u^{3 / 2}(|\log u|+1)^{3 / 2} \psi d x\right\}^{2 / 3} \\
& \leq \int_{\Omega} u^{2} \psi d x+d_{3 / 2,3 / 2}|\Omega|+\frac{4}{27} B^{3}\|v\|_{3}^{3} .
\end{aligned}
$$

Finally, III, defined by (4.1), is estimated as follows:

$$
\begin{aligned}
& I I I \leq M_{1} \int_{\partial \Omega} u \psi d S \leq M_{1} M_{2}\left(\int_{\Omega} u(\psi+|\nabla \psi|) d x+\int_{\Omega}|\nabla u| \psi d x\right) \\
& \leq M_{1} M_{2}\left(\|u\|_{1}+\int_{\Omega} u \psi^{1 / 2} \cdot A \psi^{1 / 3} d x+\int_{\Omega} u^{-1 / 2}|\nabla u| \cdot u^{1 / 2} \psi d x\right) \\
& \leq \frac{1}{4} \int_{\Omega} u^{-1}|\nabla u|^{2} d x+\int_{\Omega} u^{2} \psi d x+C_{7},
\end{aligned}
$$

where $M_{1}$ and $M_{2}$ are positive constants determined by (3.17) and (3.18), and $C_{7}$ is an constant depending only on $\left\|u_{0}\right\|_{1}, M_{1}, M_{2}$ and $A$. Combining (4.2)(4.6), we get the desired estimate.

We are now in a position to prove the finiteness of blowup points.

Proof of Theorem 3. We take $x_{0} \in \mathcal{B}$. From Lemmas 5 and 8 , it follows that

$$
\frac{d}{d t} \int_{\Omega} u \log u \psi d x+\frac{1}{4}\left(1-40 K^{2} \int_{B_{R}\left(x_{0}\right) \cap \Omega} u d x\right) \int_{\Omega} u^{-1}|\nabla u|^{2} \psi d x \leq C_{9},
$$

and therefore, the condition

$$
\limsup _{t \uparrow T} \int_{B_{R}\left(x_{0}\right) \cap \Omega} u d x<\varepsilon_{0}:=\frac{1}{40 K^{2}}
$$


implies

$$
\underset{t \uparrow T}{\limsup } \int_{B_{R}^{\prime}\left(x_{0}\right) \cap \Omega} u \log u d x \leq \limsup _{t \uparrow T} \int_{\Omega}(u \log u) \psi d x<\infty
$$

for any $0<R^{\prime}<R$. This implies $x_{0} \notin \mathcal{B}$ by Lemma 6 , a contradiction. That is, each $x_{0} \in \mathcal{B}$ admits the estimate

$$
\limsup _{t \uparrow T} \int_{B_{R}\left(x_{0}\right) \cap \Omega} u d x \geq \varepsilon_{0} \quad \text { for any } 0<R \ll 1 .
$$

We shall replace this inequality by

$$
\liminf _{t \uparrow T} \int_{B_{R}\left(x_{0}\right) \cap \Omega} u d x \geq \varepsilon_{0} \quad \text { for any } 0<R \ll 1 .
$$

If this is the case, it follows that

$$
\sharp \mathcal{B} \leq \frac{\left\|u_{0}\right\|_{1}}{\varepsilon_{0}}<\infty
$$

by virtue of the mass conservation (3.2), and then the proof is complete.

To prove $(4.10)$, it suffices to show that

$$
\sup _{0<t<T}\left|\frac{d}{d t} \int_{\Omega} u \psi d x\right| \leq C\left(x_{0}\right)<\infty
$$

by the assumption $T_{\max }<+\infty$. In fact, if (4.11) is the case, the convergence

$$
\lim _{t \uparrow T} \int_{\Omega} u \psi d x=\int_{\Omega} u_{0}(x) \psi(x) d x+\int_{0}^{T}\left(\frac{d}{d t} \int_{\Omega} u(\cdot, t) \psi d x\right) d t
$$

exists, and therefore it holds that

$$
\liminf _{t \uparrow T} \int_{B_{R}\left(x_{0}\right) \cap \Omega} u d x \geq \lim _{t \uparrow T} \int_{\Omega} u \psi d x \geq \limsup _{t \uparrow T} \int_{B_{R}^{\prime}\left(x_{0}\right) \cap \Omega} u d x \geq \varepsilon_{0}
$$

because $0<R \ll 1$ is arbitrary in (4.9).

Now, we use the first equation of $(\mathrm{GCZ})$ and $\left.\frac{\partial \psi}{\partial \nu}\right|_{\partial \Omega}=0$, and derive

$$
\frac{d}{d t} \int_{\Omega} u \psi d x=\int_{\Omega} u \Delta \psi d x+\int_{\Omega} u \nabla v \cdot \nabla \psi d x=: F_{3}+F_{4} .
$$

It is clear that

$$
\left|F_{3}\right| \leq B\left\|u_{0}\right\|_{1}
$$


Also, $F_{4}$ is equal to

$$
\begin{aligned}
& \int_{\Omega} u(x, t) \nabla \psi(x) \cdot \nabla_{x}\left\{\int_{\Omega} G\left(x, x^{\prime}\right)\left[u\left(x^{\prime}, t\right)-f\left(v\left(x^{\prime}, t\right)\right)\right] d x^{\prime}\right. \\
& \left.-\int_{\partial \Omega} G(x, \xi) g(v(\xi, t)) d S_{\xi}\right\} d x \\
& =\frac{1}{2} \int_{\Omega} \int_{\Omega} \rho\left(x, x^{\prime}\right) u(x, t) u\left(x^{\prime}, t\right) d x d x^{\prime} \\
& -\int_{\Omega} \int_{\Omega} u(x, t) \nabla \psi(x) \cdot \nabla_{x} G\left(x, x^{\prime}\right) f\left(v\left(x^{\prime}, t\right)\right) d x^{\prime} d x \\
& -\int_{\Omega} u(x, t) \nabla \psi(x) \cdot\left\{\int_{\partial \Omega} \nabla_{x} G(x, \xi) g(v(\xi, t)) d S_{\xi}\right\} d x \\
& =: F_{4}^{(1)}-F_{4}^{(2)}-F_{4}^{(3)} .
\end{aligned}
$$

Lemma 7 implies that

$$
\left|F_{4}^{(1)}\right| \leq\|\rho\|_{\infty}\left\|u_{0}\right\|_{1}^{2}
$$

while

$$
\begin{aligned}
& \left|F_{4}^{(2)}\right| \leq A\left\|u_{0}\right\|_{1}\left\|(-\Delta+1)^{-1} f(v)\right\|_{W^{1, \infty}} \leq C_{10}\left\|(-\Delta+1)^{-1} f(v)\right\|_{W^{2,3}} \\
& \leq C_{11}\|f(v)\|_{3} \leq C_{12}\left(1+\|v\|_{3 p_{1}}^{p_{1}}\right) \leq C_{13}
\end{aligned}
$$

by the Sobolev embedding, (1.7) and (3.4), where $C_{13}$ is independent of $t$. Finally, if $\psi$ is supported in $\bar{\Omega}$ it holds that

$$
\left|F_{4}^{(3)}\right| \leq C_{15}\left(x_{0}\right)
$$

which is the case of $x_{0} \in \Omega$. We obtain the finiteness of the inner blowup points and the proof is complete.

The finiteness of boundary blowup points is an open qustion. In this connection, we recall that this situation is also the case of the Dirichlet boundary condition to $v$ where the non-existence of the boundary blowup point arises in the stationary state, see [18].

The crucial estimate in our argument arises in accordance with

$$
H=\int_{\partial \Omega} G(x, \xi) g(v(\xi, t)) d S_{\xi}
$$

satisfying

$$
(-\Delta+1) H=0 \text { in } \Omega, \quad \frac{\partial H}{\partial \nu}=g \text { on } \partial \Omega
$$


for $g(\xi, t)=g(v(\xi, t))$. An expected sharp estimate is concerned with the weak norm, see [10],

$$
\|v\|_{H_{w}^{1}(\Omega)} \leq C
$$

in (2.1) for the prescribed $\|u\|_{1}=\lambda$. Then, it will follow that

$$
\|g\|_{H_{w}^{1}(\Omega)} \leq C
$$

if $g=g(v)$ is appropriate. Then, we take $\zeta \in H_{w}^{2}(\Omega)$ such that $\frac{\partial \zeta}{\partial \nu}=g$ on $\partial \Omega$. Then we obtain

$$
(-\Delta+1) h=(\Delta-1) \zeta \text { in } \Omega, \quad \frac{\partial h}{\partial \nu}=0 \text { on } \partial \Omega
$$

for $h=H-\zeta$ with $(\Delta-1) \zeta \in L_{w}^{2}(\Omega)$. This relation will imply

$$
\|H\|_{H_{w}^{2}(\Omega)} \leq C,
$$

and consequently,

$$
\left|\int_{\Omega} u \nabla \psi \cdot \nabla H d x\right| \leq C \int_{\Omega}\left(u \log u+e^{-1}\right) d x
$$

by

$$
\begin{aligned}
& \left|\int_{\Omega} u \nabla \psi \cdot \nabla H d x\right| \leq C[u]_{L \log L}\|H\|_{H_{w}^{2}(\Omega)} \\
& {[f]_{L \log L}=\int_{\Omega}|f(x)| \log \left(1+\frac{|f(x)|}{\|f\|_{1}}\right) d x .}
\end{aligned}
$$

Thus we obtain

$$
\left|\frac{d}{d t} \int_{\Omega} u \psi d x\right| \leq C\left(1+\int_{\Omega}\left(u \log u+e^{-1}\right) \psi d x\right)
$$

besides (4.8).

Inequality (4.18) is not appropriate to control the local $L^{1}$ norm of $u$ in time, but we can infer the finiteness of boundary blowup points, assuming

$$
\iint_{\Omega \times[0, T)} u \log u d x d t<+\infty .
$$

In this connection, we remind

$$
\int_{\Omega} u \log u d x \geq \delta_{1} \log \frac{1}{T-t}-C
$$

derived from (3.39) with $\delta_{1}>0$. 


\section{References}

[1] R.A. Adams, Sobolev Spaces, Academic Press (1975).

[2] S. Agmon, A. Douglis and L. Nirenberg, Estimates near the boundary for solution of elliptic partial differential equations satisfying general boundary conditions I, Comm.Pure.Appl.Math., 12 (1959), 623-727.

[3] N.D. Alikakos, $L^{p}$ bounds of solutions of reaction-diffusion equations, Comm. Partial Differential Equations, 4 (1979), 827-868.

[4] P. Biler, Existence and asymptotics for a parabolic-elliptic system with nonlinear no-flux boundary conditions, Nonlinear Anal. TMA, 19 (1992), 1121-1136.

[5] P. Biler, Local and global solvability of some parabolic systems modelling chemotaxis, Adv. Math. Sci. Appl.,8 (1998), 715-743.

[6] P. Biler and T. Nadzieia, Existence and nonexistence of solutions for a model of gravitational in interaction of particles, I.Colloq.Math., 66 (1994), 319-334.

[7] H. Brezis and W.A. Strauss, Semilinear second-order elliptic equation in $L^{1}$, J.Math.Soc.Japan, 25 (1973), 565-590.

[8] H. Chen and X.-H. Zhong, Global existence and blow-up for the solutions to nonlinear parabolic-elliptic system modelling chemotaxis, IMA J.Appl.Math., 70 (2005), 221-240.

[9] S. Childress and J.K. Percus, Nonlinear aspects of chemotaxis, Math.Biosci., 56 (1981), 217-237.

[10] G.B. Folland, Real Analysis, Modern Techniques and their Applications, John Wiley and Sons, Inc., New York (1984).

[11] H. Gajewski and K. Zacharias, Global behaviour of a reaction-diffusion system modelling chemotaxis, Math. Nachr., 195 (1998), 77-114.

[12] D. Gilbarg and N.S. Trudinger, Elliptic Partial Differential Equations of Second Order, second ed., Springer-Verlag, Berlin (1983).

[13] M. Kurokiba and T. Suzuki, On a perturbed system of chemotaxis, International J. Mathematical Analysis, 1 (2007), 1261-1272. 
[14] O.A. Ladyžhenskaja, V.A. Solonikov, and N.N. Ural'ceva, Linear and Quasilinear Equations of Parabolic Type, Amer. Math. Soc. Translations. vol.23, Amer. Math. Soc., Providence, R. I. (1968).

[15] J.L. Lions, Quelques Méthodes de Résolution de Problèmes aux Limites Non Linéaires, Dunod-Gauthier-Villas, Paris (1969).

[16] T. Nagai, Blow-up of radially symmetric solutions to a chemotaxis, Adv. Math. Sci. Appl., 5 (1995), 581-601.

[17] T. Nagai, T. Senba and K. Yoshida, Application of the Trudinger-Moser inequality to a parabolic system of chemotaxis, Funkcialaj Ekvacioj, 40 (1997), 411-433.

[18] K. Nagasaki and T. Suzuki, Asymptotic analysis for two-dimensional elliptic eigenvalue problems with exponentially dominated nonlinearities, Asymptotic Analysis, 3 (1990), 173-188.

[19] V. Nanjundiah, Chemotaxis, signal relaying, and aggregation morphology, J. Theor. Biol., 42 (1973), 63-105.

[20] M.H. Protter and H.F. Weinberger, Maximum Principles in Differential Equations, Springer-Verlag, New York (1984).

[21] T. Senba and T. Suzuki, Chemotactic Collapse in a parabolic-elliptic system of mathematical biology, Adv.Diff.Eqns, 6 (2001), 21-50.

[22] T. Senba and T. Suzuki, Parabolic system of chemotaxis; blowup in a finite and in the infinite time, Meth. Appl. Anal.,8 (2001), 349-368.

[23] T. Suzuki, Free Energy and Self-Interacting Particles, Birkhäuser Verlag, Boston (2005). 